\title{
Clinical performance of the levonorgestrel intrauterine system in routine use by the UK Family Planning and Reproductive Health Research Network*: 5-year report
}

\author{
Michael Cox, FRCOG, MFFP, Consultant Obstetrician and Gynaecologist (Retired), Nuneaton, UK; John Tripp, MD, FRCPCH, \\ School of Postgraduate Medicine and Health Sciences, University of Exeter, UK; Sarah Blacksell, BA, Research Fellow, School \\ of Postgraduate Medicine and Health Sciences, University of Exeter, UK
}

Correspondence: UK Family Planning and Reproductive Health Research Network, c/o John Tripp, Department of Child Health, Church Lane, Heavitree, Exeter EX2 5SQ, UK

\begin{abstract}
*Principal investigators: A Barnett (Exeter), P Barnes (Richmond, London), J Bateman (Portsmouth), J Bland (Nuneaton), D Booker (Newport), R Bradbury (Barnsley), S Brown (Birmingham), G Cardy (Bristol), S Carr (Glasgow), H Cooling (Bristol), $M$ Cox (Nuneaton), D Craggs (Southampton), J Dewsbury (Birmingham), J Elstub (Richmond, London), S Green (Cumbria), B Hanson (Wiltshire), T Laverty (Wiltshire), A Main (Richmond, London), H Massil (London CHSL), C Nash (Norwich), E Oloto (Leeds), R Owen (Taunton), S Randall (Portsmouth), S Richardson (West Yorkshire), S Rowlands (Biggleswade), J Rumsey (Birmingham), C Smith (Thetford), J Tattersall (Cumbria), A Taylor(Sheffield), A Thomas (Newport), R Tolcher (Southampton), C Tupper (Cumbria), A Turner (Wiltshire), $M$ Walling (Boston), C Watson (London CHSL), E M Watt (Bristol)
\end{abstract}

(Accepted $26^{\text {th }}$ February 2002)

The Journal of Family Planning and Reproductive Health Care 2002: 28(2): 73-77

\begin{abstract}
Objectives. This study was undertaken to determine the performance of the levonorgestrel intrauterine system (LNG IUS) in British women in routine clinical use.

Design. Doctors working in general practice and at family planning clinics throughout the UK who collaborate in the UK Family Planning and Reproductive Health Research Network were responsible for the fitting of 678 LNG IUSs. Results. Gross cumulative event rates at 5 years per 100 women were pregnancy 1.0, expulsion 5.9, removal for bleeding problems 16.7, removal for pain 4.3, and removal for pelvic inflammatory disease (PID) 1.2. Removals were also required for side effects that may be due to absorbed levonorgestrel. Positive effects include effective contraception and considerably reduced quantity of bleeding for most participants.

Conclusions. A need was identified to counsel women about the early bleeding problems including the possibility of oligomenorrhoea or amenorrhoea. This is considered to be very important as it will help women to persevere so that they can enjoy the longer-term benefits.
\end{abstract}

\section{Key message points}

- At 5 years of use the LNG IUS (Mirena ${ }^{\circledR}$ ) has been shown to have a very low pregnancy rate and a low expulsion rate.

- The rate for removal for complaints of bleeding problems was high during the first year.

- There is a need for careful counselling before fitting to prepare women for changes in bleeding patterns during the first 12 months of use.

- Removals were also required for side effects that may be due to absorbed levonorgestrel.

- The reductions in duration and quantity of periods in many women were significant advantages.

\section{Introduction}

This report concludes the study of which the 12-month and 24-month results have been published. ${ }^{1,2}$ The concept of intrauterine administration of a progestin-only contraceptive as an alternative to oral, parenteral or subdermal implants was first considered in $1970 .^{3}$ Experiments with various slow-release membranes and intrauterine contraceptive device (IUD)-carrying frames resulted in the development of an intrauterine system (IUS) releasing levonorgestrel, now marketed as Mirena ${ }^{\circledR}$ which was introduced in Finland in 1990 and in the UK in May 1995.

Very low pregnancy rates have been reported, between 0.5 and 1.1 cumulative gross rates per 100 users at 5 years $^{4-6}$ (Table 1). Expulsion rates have been similar to that of other IUDs. The Mirena ${ }^{\circledR}$ is recommended for use up to 5 years, although very low pregnancy rates have also been reported after up to 7 years of use. ${ }^{7}$

The endometrium remains atrophic while the levonorgestrel is at a sufficient level to suppress it, ${ }^{8}$ and therefore changes in menstrual patterns are likely to occur. Sivin and Stern, ${ }^{9}$ reporting a multicentre study, commented that 'menstrual patterns during contraception were radically altered by the LNG'.

Several randomised trials have found that discontinuation rates because of heavy or prolonged menstrual blood flow were significantly lower with the LNG IUS than with copper IUDs. ${ }^{10}$ Sivin et al. ${ }^{11}$ found that discontinuation attributable to amenorrhoea was the principal contributor to differences in continuation rates between the LNG IUS and the Cu T380A ${ }^{\circledR}$ IUD.

There is a need to determine the performance of the LNG IUS in British women with particular regard to the nature and incidence of side effects.

\section{Methods}

The study was an open, single group, multicentre phase III study in outpatients. Doctors working in general practice and at family planning clinics throughout the UK who cooperate in the UK Family Planning and Reproductive Health Research Network were responsible for the LNG IUS fittings reported in this paper. The study was sponsored 
Table 1 The LNG IUS: cumulative gross closure rates at 5 years per 100 users

\begin{tabular}{|c|c|c|c|c|c|c|c|c|c|c|c|}
\hline Study & Location & $\mathrm{n}$ & Pregnancy & Expulsion & $\begin{array}{l}\text { Bleeding } \\
\text { problems }\end{array}$ & Pain & Amenorrhoea & Hormonal & PID & $\begin{array}{l}\text { Other } \\
\text { medical }\end{array}$ & $\begin{array}{l}\text { Continuation } \\
\text { rate }\end{array}$ \\
\hline Andersson et al. (1994) & Scandinavia & 1821 & 0.5 & 5.8 & 13.7 & 5.9 & 6.0 & 12.1 & 0.8 & 7.7 & 55.5 \\
\hline Luukkainen et al. $(1986)^{5}$ & Finland & 281 & 0.8 & 2.0 & $8.3^{\mathrm{a}}$ & - & 12.8 & 9.5 & 1.3 & 4.1 & 53 \\
\hline \multirow[t]{2}{*}{ Sivin et al. ${ }^{c}(1990)^{6}$} & Egypt, South & & & & & & & & & & \\
\hline & America & 1124 & 1.1 & 11.8 & 15.4 & - & 19.7 & - & 2.6 & 16.9 & 33 \\
\hline Present study & UK & 678 & 1.0 & 5.9 & 16.7 & 4.3 & - & - & 1.2 & $20.8^{\mathrm{b}}$ & 39.6 \\
\hline
\end{tabular}

${ }^{a}$ Includes pain.

bIncludes amenorrhoea and hormonal.

${ }^{\mathrm{c}}$ There were five perforations in the Sivin study but none in the others.

PID, Pelvic inflammatory disease.

by Leiras Oy and Schering Health Care Limited. Ethics committee approval for the study was obtained in all centres.

The system consists of a T-shaped polyethylene frame carrying a hormone cylinder containing $52 \mathrm{mg}$ of levonorgestrel. The cylinder is covered with a polydimethylsiloxane membrane that regulates the release of $20 \mu \mathrm{g}$ levonorgestrel per day.

The system was fitted according to the doctor's own clinical judgement, in parous women attending for family planning advice and requesting intrauterine contraception. Those enrolled were parous women aged 18-45 years, exposed to the risk of pregnancy, in good health and with normal menstrual cycles. They were fitted with their IUS more than 6 weeks after delivery. After entry to the study, the subjects were followed for 5 years.

After insertion of the IUS, each subject was requested to return for follow-up examination at 3 months, 12 months and annually thereafter. Baseline data and details of the fitting procedure and follow-up visits were forwarded to the study co-ordinator at the University of Exeter, where the data were processed and analysed. Event rates were calculated using the life-table method. ${ }^{12}$ Adverse events were classified using the WHO Adverse Reaction Dictionary (Version 1/1995).

A programme of comprehensive monitoring using EU Good Clinical Practice (GCP) Guidelines was used. The study commenced in July 1992. At the close of recruitment in December 1995, 678 women had been recruited who met the criteria of the study protocol (reasons for protocol violation are listed in Table 2). This paper reports the data from this per protocol sample for 5 years of use. Some minor adjustments to the data and sample occurred as a result of monitoring during the later stages of the study and also the use of additional methods of contraception by some subjects during the course of the study ( 21 removals from the sample, seven reinstatements). The use of additional hormonal methods of

Table 2 Reasons for protocol violation $(n=111)$

\begin{tabular}{lr}
\hline Reason & $\mathrm{n}$ \\
\hline Over 45 years of age & 8 \\
Nulliparous & 22 \\
Second or subsequent fitting & 12 \\
IUS fitted as emergency contraception & 4 \\
Pregnant at fitting & 1 \\
Fitting at less than 6 weeks since live birth & 3 \\
Concomitant method; using POP for more than one cycle & 10 \\
Concomitant method; using COC or HRT & 37 \\
Concomitant method; subject using other methods & 14 \\
(pessaries, spermicides) &
\end{tabular}

COC, Combined oral contraceptive; HRT, hormone replacement therapy; POP, progestogen-only pill. contraception was a protocol violation for this study. As a result, the sample differs slightly from the sample used in the previous papers on 12 and 24 months of use. ${ }^{1,2}$

\section{Results}

Demographic characteristics

The mean age of the study population was 33 years (range 18-45 years). All the women were parous: $70.4 \%$ had given birth to one or two children and $29.5 \%$ had given birth to three or more. The age distribution is shown in Table 3.

Table 3 Age distribution

\begin{tabular}{lrr}
\hline Age group (years) & $\mathrm{n}$ & $\%$ \\
\hline $18-24$ & 69 & 10 \\
$25-29$ & 145 & 21 \\
$30-34$ & 168 & 25 \\
$35-39$ & 188 & 28 \\
$40-45$ & 108 & 16 \\
Total & 678 & 100 \\
\hline
\end{tabular}

\section{Cumulative life-table gross closure rates}

The gross cumulative rates for closure of study per 100 women at 12, 24, 36, 48, and 60 months are given in Table 4 . At 5 years of use, the pregnancy rate was very low [1.0, 95\% confidence interval (CI) $0.3-2.4]$. The expulsion rate was also low $(5.9,95 \%$ CI 3.9-7.9). There were no pregnancies and only one expulsion after 24 months. The rate for removal for bleeding problems and bleeding with pain was high during the first year $(10.5,95 \%$ CI $8.0-13.1)$ and was 16.7 (95\% CI 13.3-20.0) at 5 years. The rate for removals for other complaints seen to be associated with IUS use was 20.8 (95\% CI 17.0-24.7) at 5 years.

\section{Pregnancy}

The gross cumulative rate for pregnancy was 1.0 at 5 years. Three pregnancies occurred during the first 12 months of which two resulted in spontaneous abortion and one in a missed abortion. Two more pregnancies occurred between 12 and 24 months, one being an ectopic and one resulting in a normal pregnancy and delivery. The three pregnancies occurring during the first 12 months have been described in the 12-month report. ${ }^{1}$ The other two are described below.

Case report 1. Pregnancy occurred after 14 months of use. An ultrasound examination at 16 months failed to show the IUS so it was presumed to have been expelled prior to conception. The subject wished to continue with the pregnancy, which proceeded normally with a normal delivery of a live infant.

Case report 2. This case has been reported previously. ${ }^{13}$ The ectopic pregnancy was treated at 15 months of use by salpingectomy and the IUS was removed. 
Table 4 Cumulative life-table gross closure rates per 100 users at 12,24, 36, 48 and 60 months (per protocol sample of 678)

\begin{tabular}{|c|c|c|c|c|c|c|c|c|c|c|c|c|c|c|c|}
\hline & \multicolumn{3}{|c|}{12 months } & \multicolumn{3}{|c|}{24 months } & \multicolumn{3}{|c|}{36 months } & \multicolumn{3}{|c|}{48 months } & \multicolumn{3}{|c|}{60 months } \\
\hline & $\mathrm{n}$ & GR & CI & $\mathrm{n}$ & GR & CI & $\mathrm{n}$ & GR & CI & $\mathrm{n}$ & GR & CI & $\mathrm{n}$ & GR & CI \\
\hline Pregnancy & 3 & 0.6 & $0.1-1.6$ & 5 & 1.0 & $0.3-2.4$ & 5 & 1.0 & $0.3-2.4$ & 5 & 1.0 & $0.3-2.4$ & 5 & 1.0 & $0.3-2.4$ \\
\hline $\begin{array}{l}\text { Expulsion } \\
\text { Side effects }\end{array}$ & 27 & 4.5 & $2.8-6.2$ & 30 & 5.2 & $3.3-7.0$ & 31 & 5.5 & $3.6-7.4$ & 31 & 5.5 & $3.6-7.4$ & 32 & 5.9 & 3.9-79 \\
\hline Perforation & 0 & 0.0 & $0.0-0.0$ & 0 & 0.0 & $0.0-0.0$ & 0 & 0.0 & $0.0-0.0$ & 0 & 0.0 & $0.0-0.0$ & 0 & 0.0 & $0.0-0.0$ \\
\hline Bleeding problems & 62 & 10.5 & $8.0-13.1$ & 71 & 12.6 & $9.8-15.4$ & 75 & 13.7 & $10.8-16.7$ & 78 & 14.7 & $11.6-17.8$ & 83 & 16.7 & $13.3-20.0$ \\
\hline Pain & 13 & 2.3 & $1.0-3.5$ & 18 & 3.5 & $1.9-5.2$ & 18 & 3.5 & $1.9-5.2$ & 20 & 4.3 & $2.4-6.2$ & 20 & 4.3 & $2.4-6.2$ \\
\hline PID & 5 & 0.9 & $0.3-2.0$ & 6 & 1.2 & $0.4-2.5$ & 6 & 1.2 & $0.4-2.5$ & 6 & 1.2 & $0.4-2.5$ & 6 & 1.2 & $0.4-2.5$ \\
\hline $\begin{array}{l}\text { Other complaint } \\
\text { associated with }\end{array}$ & & & & & & & & & & & & & & & \\
\hline $\begin{array}{l}\text { IUS use } \\
\text { Not related }\end{array}$ & 42 & 7.7 & $5.4-9.9$ & 63 & 12.4 & $9.5-15.3$ & 81 & 17.3 & $13.8-20.8$ & 91 & 20.4 & $16.6-24.3$ & 92 & 20.8 & $17.0-24.7$ \\
\hline $\begin{array}{l}\text { Planning pregnancy } \\
\text { Medical not related }\end{array}$ & 16 & 3.2 & $1.6-4.8$ & 33 & 7.4 & $4.9-9.9$ & 45 & 10.9 & $7.8-14.0$ & 54 & 13.9 & $10.4-17.4$ & 55 & 14.3 & $10.7-17.9$ \\
\hline to IUS use & 10 & 1.9 & $0.9-3.4$ & 11 & 2.2 & $0.9-3.5$ & 12 & 2.5 & $1.1-3.9$ & 14 & 3.3 & $1.5-5.0$ & 16 & 4.2 & $2.1-6.2$ \\
\hline Other reason & 18 & 3.3 & $1.8-4.8$ & 32 & 6.8 & $4.5-9.1$ & 41 & 9.4 & $6.6-12.1$ & 46 & 11.1 & $8.0-14.1$ & 56 & 15.0 & $11.3-18.7$ \\
\hline Total closures & 196 & & & 269 & & & 314 & & & 345 & & & 365 & & \\
\hline $\begin{array}{l}\text { Continuation with } \\
\text { device (net rate) } \\
\text { Lost to follow-up }\end{array}$ & 45 & 69.6 & & 65 & 57.4 & & 78 & 49.5 & & 92 & 43.7 & & 96 & 39.6 & \\
\hline
\end{tabular}

CI, 95\% Confidence intervals; GR, gross rate; IUS; intrauterine system; n, number; PID, pelvic inflammatory disease.

\section{IUS removal following a complaint of bleeding or bleeding} with pain

A total of 83 women had the system removed at or before 5 years following a complaint of bleeding problems or of bleeding with pain, giving a gross cumulative rate of 16.7 at 5 years (Table 4). The average length of use before removal of the system for these reasons was 11.7 months. The type of complaint leading to removal of the system following a complaint of bleeding or bleeding and pain is shown in Table 5 . In more than half of these cases $(43 / 83 ; 52 \%)$ the subject complained of continuous or persistent vaginal loss. An additional 17 women originally in the study had their bleeding problems treated with hormones and then had to be withdrawn from the sample as this contradicted the protocol.

Table 5 Bleeding problems leading to IUS removal at or before 5 years of use

\begin{tabular}{lrr}
\hline Reason for removal & $\mathrm{n}$ & $\%$ \\
\hline Continuous/persistent vaginal loss & 43 & 52 \\
Irregular bleeding & 18 & 21 \\
Heavier periods/‘bleeding' & 13 & 16 \\
Longer periods & 9 & 11 \\
Total & 83 & 100 \\
\hline
\end{tabular}

Other medical complaints requiring IUS removal

A total of 92 women had the device removed at or before 5 years following medical complaints associated with the IUS, giving a rate of 20.8 (Table 4). Since in some cases these were described as groups of symptoms, with no one principal reason for removal, these are therefore listed in Table 6 by frequency of occurrence. Within this category, the majority of removals were due to a range of symptoms, some of which may be related to progestogen use; this is discussed below.

The largest group in this category is oligo/amenorrhoea (26 complaints). In this group, amenorrhoea was the majority with 18 complaints, of which six were of amenorrhoea alone and 12 of amenorrhoea together with other complaints. Oligo/amenorrhoea was not included in the category of 'bleeding problems' in this study. This makes comparison with some other studies difficult (Table 1).
Table 6 Medical reasons for removal by 5 years of use (considered to be possibly associated with the IUS) in 92 subjects, some having more than one complaint

\begin{tabular}{llll}
\hline Complaint & $\mathrm{n}$ & Complaint & $\mathrm{n}$ \\
\hline Oligo/amenorrhoea & 26 & Dizziness & 3 \\
Weight gain & 16 & Candida & 3 \\
Premenstrual-type symptoms/ & & Dyspareunia (female) & 3 \\
PMT & 14 & Postcoital bleeding & 3 \\
Mood swings/depression & 13 & Hirsutism & 3 \\
Bloating & 13 & Conscious of IUS & 3 \\
Breast tenderness & 12 & Ovarian cyst & 2 \\
Headaches/migraine & 9 & Palpitations & 2 \\
Vaginal discharge & 8 & Dyspareunia (male) & 2 \\
Acne & 7 & Offensive smell & 2 \\
Abdominal pain/cramps & 6 & Thinning hair & 2 \\
Tiredness/felt unwell & 6 & Prolonged bleeding & 2 \\
Loss of libido & 5 & Breast nodularity & 2 \\
Nausea & 4 & Other & $13^{\mathrm{a}}$ \\
\end{tabular}

ancludes the following (one complaint each): mastalgia; hot sweats; leg pain; burning vagina; back ache; cervicitis; dysmenorrhoea; vaso-vagal attack after fitting; septicaemia; exacerbation of pre-existing ulcerative colitis and acne rosacea; neurological symptoms/jaundice/allergic reaction.

Other reasons for IUS removal

A total of 56 women had the device removed at or before 5 years for various other reasons as shown in Table 7 .

\section{Serious adverse events}

(a) Pelvic inflammatory disease (PID). The diagnosis depended on the judgement of the clinician involved. The gross cumulative rate for removal for PID was 1.2 at 5 years (Table 4). Six systems were removed for PID, though the diagnosis was unproven in three. There was one other case in which the system was not removed (Table 8). The relationship of PID to the LNG IUS is uncertain and is discussed below. (One subject recorded as a case of PID in the 12-month report was found at a later monitoring visit to have used another form of contraception, and therefore is a protocol violator and not included in the present sample.) (b) Deaths and other serious adverse events. Two accidental deaths occurred before 12 months of use, one by drowning and one in a fire. The following cases also occurred: 
Table 7 Other reason for IUS removal

\begin{tabular}{lrr}
\hline Reason for removal & $\mathrm{n}$ & $\%$ \\
\hline Sterilisation/partner vasectomy & 21 & 38 \\
Subject's request $^{\mathrm{a}}$ & 13 & 23 \\
IUS no longer needed (partner death/end of relationship) $^{\text {Accidental removal by subject }}$ & 10 & 18 \\
Investigator's decision $^{\text {b }}$ & 4 & 7 \\
Reason not known $_{\text {Total }}$ & 4 & 7 \\
& 56 & 7 \\
\hline
\end{tabular}

aSubject's request: 'unhappy' with IUS (4); fear of hormones (3); loss of confidence (because of amenorrhoea, fear of losing threads, "unsafe after 3 years') (3); reason not known (2); moving abroad (1).

'Investigator's decision: lost threads; subject's history of stroke; subject menopausal; false pregnancy test.

(i) Age 29. Ruptured ovarian cyst four months after fitting. Right salpingo-oophorectomy was performed. The LNG IUS was not removed. This subject is also the first in Table 8 with PID which occurred 1 month later.

(ii) Age 37. Abdominal pain due to ovarian cyst 7 months after fitting. Ovarian cystectomy was performed.

(iii) Age 33. 'Inflammation of the bowel' 5 days after fitting. Hospitalised. Septicaemia. Haemolytic streptococcus on blood culture. IUS removed from uterus. History of Crohn's disease. The surgeon thought the IUS may be the cause, the gynaecologist thought the Crohn's to be responsible.

(iv) Age 43. Forty months after fitting developed severe cluster headaches and left ptosis. Extensive neurological investigation. No definite diagnosis. Treated with corticosteroids. Recovered. One further attack 6 months later for 1 day. The principal investigator thought the IUS was possibly related but it was not removed.

(v) Age 38. Exacerbation of pre-existing ulcerative colitis and acne rosacea 25 months after fitting. IUS removed. Outcome after removal not known.

(vi) Age 37. Eight months after fitting developed optic neuritis, thought unlikely to be related to the IUS. Eleven months after fitting developed mild jaundice, anaemia and raised bilirubin. Thought to be possibly related to the IUS. Twenty-four months after fitting severe allergic reaction to Redoxon. IUS was removed.

(c) Ovarian cysts. Fifteen subjects developed an ovarian cyst with a further two suspected cases. The IUS was removed in four cases, and in a fifth a ruptured cyst was removed with right salpingo-oophorectomy [Case (i) above]. A cyst was removed in two further cases, and in one of these cases the IUS was removed 3 months later after heavy bleeding and partial expulsion of the device.

\section{Discussion}

Fitting experience shows that there appears to be a learning curve as most failed fittings were in the early months. This has been reported in more detail elsewhere. ${ }^{14}$
The gross cumulative rates at 5 years are compared to three other published studies in Table 1. All four studies show very low pregnancy rates, between 0.5 and 1.1 . Of the five pregnancies that occurred in the present study, two resulted in spontaneous abortions, one in a missed abortion, one was ectopic, and one pregnancy proceeded normally. All the pregnancies occurred in the first 24 months. It has long been known that the spontaneous abortion rate is high when a pregnancy occurs in the presence of an IUD. ${ }^{15}$ Although the risk of ectopic pregnancy is extremely low with the LNG IUS, it can nevertheless occur, as in the present study and others. ${ }^{16-18}$ The expulsion rate and the removal for PID rates were low and there were no perforations.

The most common side effect resulting in removal was bleeding problems, analysed in Table 5. Most cases (62/83) occurred during the first 12 months. There is a need to be aware of a cultural aspect of this problem; a Muslim woman requested removal because prolonged bleeding interfered with prayer. Unfortunately it is difficult to make accurate comparisons with other studies because there has been a variation in the way in which bleeding, pain, amenorrhoea, hormonal effects and other medical reasons are classified (Table 1).

Oligomenorrhoea or amenorrhoea are more evident after the first 12 months. (In the present study oligomenorrhoea and amenorrhoea were not classified separately.) Whether these changes are considered as positive or negative will vary between individuals. Many women in the present study commented favourably on the reduction of duration and quantity of menstrual bleeding. This reduction is such that the LNG IUS is finding an important place in the treatment of excessive uterine bleeding. ${ }^{19-26}$ The LNG IUS has been licensed in the UK for the treatment of menorrhagia since January 2001.

There is therefore a need to counsel women about the early bleeding problems including the possibility of oligomenorrhoea or amenorrhoea. This is considered to be very important as it will help women to persevere so that they can enjoy the longer-term benefits. In the present study all investigators carried out such counselling.

Side effects, other than bleeding disturbances, may be due to levonorgestrel absorbed from the LNG IUS. Many symptoms have been associated with oral progestogens. For example, in the present study there were complaints of breast pain, weight gain, headache, depression and premenstrual tension (Table 6). The amount of levonorgestrel absorbed from an LNG IUS is very small. Are some individuals more sensitive than others to low plasma levels of levonorgestrel? Double-blind trials to give the answer are impossible. Removals for these problems were not observed in our 5-year study of the Nova T380 ${ }^{\circledR}$ IUD.

Table 8 Pelvic inflammatory disease (PID)

\begin{tabular}{|c|c|c|c|c|c|}
\hline Age (years) & $\begin{array}{l}\text { Duration of IUS } \\
\text { use (months) }\end{array}$ & Severity & Investigation/treatment & IUS removed & Result \\
\hline 29 & 4 & Severe & Hospitalisation, augmentin, metronidazole & Yes & Cured \\
\hline $29^{\mathrm{a}}$ & 5 & Mild & PID or UTI, cephadrine, metronidazole, erythromycin & Yes & Cured \\
\hline 41 & 4 & Severe & Hospitalisation, augmentin, doxycycline & Yes & Cured \\
\hline $34^{\mathrm{a}}$ & 6 & Moderate & Co-trimoxazole & Yes & Cured \\
\hline 19 & 0.75 & Moderate & Hospitalisation, antibiotics & Yes & Cured \\
\hline $31^{\mathrm{a}}$ & 22 & Not known & Hospitalisation, hysterectomy & Yes & Cured \\
\hline $31^{\mathrm{b}}$ & 2 & Not known & Flagyl & No & Cured \\
\hline
\end{tabular}

aDiagnosis doubtful.

bTreated by GP; severity not known.

IUS, Intrauterine system; UTI, urinary tract infection. 
The diagnosis of PID depended on the clinical judgement of the clinician involved since laparoscopy was not used. The seven cases of PID (Table 8) could have been related to the IUS. However the frequency of PID has been shown in recent studies to be only minimally, if at all, related to the IUD. Of greater significance is exposure to sexually transmitted infection (STI) rather than the presence of an IUD. ${ }^{27,28}$ During the period of the present study only some centres were carrying out screening and prophylactic measures for Chlamydia and other organisms. There appeared to be no increased risk of PID soon after fitting as has been reported elsewhere. ${ }^{29}$ In six of the seven cases removal of the IUS was performed.

In the present study 15 subjects developed an ovarian cyst ( $2 \%$ of 678 fittings). In a study of 1821 fittings $^{30}$ there were three removals due to ovarian cysts. In another study functional ovarian cysts were found associated with the LNG IUS surprisingly frequently: in 6/50 women. One was aspirated but the rest resolved spontaneously. ${ }^{31}$

Only $14 \%$ of patients were lost to follow-up, a proportion that is unlikely to have affected the results significantly.

\section{Conclusions}

At 5 years of use the LNG IUS has been shown to have a very low pregnancy rate and a low expulsion rate. The rate of removal for a complaint of bleeding or bleeding with pain is high, mostly during the first 12 months. Experience confirms the need for careful counselling before the system is fitted to prepare women for the changes of bleeding patterns likely to be encountered in the early months of use. Removals were also required for side effects that may be due to absorbed levonorgestrel. The reductions in duration and quantity of periods in many women were significant advantages.

\section{Acknowledgements}

The LNG IUSs were supplied by Leiras Oy. Statistical analysis was by Ruth Preist, Hilary Hardwicke and Sarah Blacksell of the School of Postgraduate Medicine, University of Exeter.

\section{Statements on funding and competing interests}

Funding. The study was sponsored by Leiras Oy and Schering Health Care Limited.

Competing interests. None declared.
References

Cox M, Blacksell S. Clinical performance of the levonorgestrel intra-uterine system in routine use by the UK Family Planning and Reproductive Health Research Network: 12 month report. Br J Fam Plann 2000; 26: 143-147.

2 Cox M, Blacksell S. Clinical performance of the levonorgestrel intra-uterine system in routine use by the UK Family Planning and Reproductive Health Research Network: 24 month report. Eur J Contracept Reprod Health Care 2000; 5: 83 .

3 Luukkainen T. The levonorgestrel-releasing IUD. Br J Fam Plann 1993; 19: 221-224.

Andersson K, Odlind V, et al. Levonorgestrel-releasing and copper releasing (NovaT) IUDs during five years of use: a randomised comparative trial. Contraception 1994; 49: 56-72.

Luukkainen T, Allonen H, Haukkamaa M, et al. Five years experience with levonorgestrelreleasing IUDs. Contraception 1986; 33: 139-148.

6 Sivin I, Mahgoub El S, McCarthy T, et al. Long term contraception with the levonorgestrel 20 $\mu \mathrm{g} / \mathrm{day}$ and the copper $\mathrm{T} 380 \mathrm{Ag}$ intrauterine devices: a five-year randomised study Contraception 1990; 42: 361-378.

7 Sivin I, Stern J, Coutinho E, et al. Prolonged intrauterine contraception: a seven year randomised study of the levonorgestrel 20 microgram/day, LNG-20 and the copper Ag IUDs. Contraception 1991; 44: 473-480.

Silverberg SG, Haukkamaa M, Arko, $\mathrm{H}$ et al. Endometrial morphology during long-term use of levonorgestrel-releasing intrauterine devices. Int J Gynecol Pathol 1986; 5(3): 235-241.

9 Sivin I, Stern J, and the International Committee for Contraception Research. Health during prolonged use of levonorgestrel 20 micrograms/day and the copper TCu $380 \mathrm{Ag}$ intrauterine contraceptive devices: a multicentre study. Fertil Steril 1994; 61: 70-77.

10 Chi I-C. The T Cu-380A (Ag), MLCu375, and Nova T IUDs and the IUD daily releasing 20 micrograms levonorgestrel - four pillars of IUD contraception for the nineties and beyond? micrograms levonorgestrel - four

11 Sivin I, et al. Prolonged intrauterine contraception: a seven-year randomised study of the levonorgestrel $20 \mathrm{mg}$ /day and the copper T380Ag IUDs. Contraception 1979; 44: 473-480.

Tietze C, Lewitt S. Recommended procedures for the statistical evaluation of intrauterine contraception. Stud Fam Plann 1973; 4(2): 35-42.

contraception. Stud Fam Plann 1973; 4(2): 35-42.

14 Tattersall J, Allison CJ, Blacksell S, in collaboration with the UK Family Planning Research Network. The levonorgestrel intrauterine system in routine use: experiences of insertion. Acta Network. The levonorgestrel intrauterine system in rout

15 Vessey MP, Johnson B, Doll R, et al. Outcome of pregnancy in women using an intrauterine device. Lancet 1974; 1: 495-498.

16 Masters T, Miskry T, Lowe D, et al. Report of ectopic pregnancies associated with the levonorgestrel intrauterine system. Br J Fam Plann 1997; 23: 25-26.

17 Gebbie A, Brown A, Pearson S. Ectopic pregnancy in a Mirena user. Br J Fam Plann 1997; 23: 69.

18 Ojutiku D, Cutner A, Rymer J. Ectopic pregnancy with levonorgestrel releasing intrauterine system. Br J Fam Plann 1998; 24: 85-8

9 Bounds W. Clinical experience with a levonorgestrel-releasing intrauterine contraceptive device as a contraceptive and in the treatment of menorrhagia. Br J Fam Plann 1993; 19 193-194

20 Sturridge F, Guillebaud J. A risk-benefit assessment of the levonorgestrel-releasing intrauterine system. Drug Safety 1996; 15(6): 430-440.

1 Royal College of Obstetricians and Gynaecologists (RCOG). The management of menorrhagia in secondary care. Evidence-based clinical guidelines, No. 5, July 1999

22 van den Hurk P, O'Brien S. Non-contraceptive use of the levonorgestrel-releasing intrauterine system. The Obstetrician \& Gynaecologist 1999; 1: 13-18.

23 Cameron IT. The levonorgestrel intrauterine system: the benefits of reduced bleeding. Eur $J$ Contracept Reprod Health Care 2001; 6 (Suppl. 1): 27-32.

24 Dolan LM, Mulholland M, Price J. The levonorgestrel intra-uterine system: Therapeutic application in family planning. J Fam Plann Reprod Health Care 2001; 27: 19-21.

25 Hurskainen R, Teperi J, Rissanen P, et al. Quality of life and cost-effectiveness of evonorgestrel-releasing intrauterine system versus hysterectomy for treatment of menorrhagia: a randomised trial. Lancet 2001; 357: 273-277.

26 Istre O, Trolle B. Treatment of menorrhagia with the levonorgestrel intrauterine system versus endometrial resection. Fertil Steril 2001; 76(2): 304-309.

Bromham DR. Intrauterine contraceptive devices - a reappraisal. Br Med Bull 1993; 49: $100-123$

28 Grimes DA. Intrauterine device and upper genital-tract infection. Lancet 2000; 356 1013-1019.

29 Farley TMH, Rosenberg MJ, Rowe P. Intrauterine devices and pelvic inflammatory disease: an international perspective. Lancet 1992; 339: 785-788.

Chi I-C. An evaluation of the levonorgestrel-releasing IUD: its advantages and disadvantages when compared to the copper releasing IUDs. Contraception 1991; 44: 573-588.

Robinson GE, Bounds W, Kubba AA, et al. Functional ovarian cysts associated with the levonorgestrel releasing intrauterine device. Br J Fam Plann 1989: 14: 131-132. 